\title{
Thompson's group $F$ is not SCY
}

\author{
Stefan Friedl and Stefano Vidussi ${ }^{1}$
}

\begin{abstract}
In this note we prove that Thompson's group $F$ cannot be the fundamental group of a symplectic 4-manifold with canonical class $K=0 \in H^{2}(M)$ by showing that its Hausmann-Weinberger invariant $q(F)$ is strictly positive.
\end{abstract}

Mathematics Subject Classification (2010). 57R17, 20F65, 57M05.

Keywords. Thompson's groups, symplectic 4-manifolds.

Symplectic 4-manifolds with trivial canonical class, oftentimes referred to as symplectic Calabi-Yau manifolds are, conjecturally, a fairly restricted class of manifolds, see $[13,5]$. Part of this restriction is reflected in known constraints for their fundamental groups, that we will refer to as $S C Y$ groups. In the case of $b_{1}>0$, these results, for which we refer to $[1,14,8]$, corroborate the expectation that such groups are (virtually) poly- $\mathbb{Z}$.

We are interested here in the following constraints, that apply to the fundamental group $G=\pi_{1}(M)$ of a symplectic Calabi-Yau 4-manifold $M$ with $b_{1}(M)=$ $b_{1}(G)>0$ :

(1) $2 \leq b_{1}(G) \leq v b_{1}(G) \leq 4$, where $v b_{1}(G)=\sup \left\{b_{1}\left(G_{i}\right) \mid G_{i} \leq f . i\right.$. $\left.G\right\}$ denotes the supremum of the first Betti number of all finite index subgroups of $G$;

(2) if the first $L^{2}$-Betti number $b_{1}^{(2)}(G)$ vanishes, then $q(G)=0$, where the Hausmann-Weinberger invariant $q(G)=\inf \left\{\chi(X) \mid \pi_{1}(X)=G\right\}$ is defined as the infimum of the Euler characteristic among all 4-manifolds whose fundamental group is $G$ ([11]).

(In [8, Proposition 2.2] the vanishing of $q(G)$ is stated under the assumption that $G$ is residually finite, but in fact only the condition $b_{1}^{(2)}(G)=0$ is used in the proof.)

${ }^{1}$ S. Vidussi was partially supported by NSF grant \#0906281. 
The purpose of this note is to apply these constraints to the case of Thompson's group $F$. The group $F$ (about which we refer to $[4,10]$ for some preliminary facts) is a group that admits the finite presentation

$$
F=\left\langle x_{0}, x_{1} \mid\left[x_{0} x_{1}^{-1}, x_{0}^{-1} x_{1} x_{0}\right]=1,\left[x_{0} x_{1}^{-1}, x_{0}^{-2} x_{1} x_{0}^{2}\right]=1\right\rangle .
$$

This group has a number of peculiar features, that make it a natural testing ground for conjectures and speculations. We should mention that S. Bauer asked (cf. [1, Question 1.5]) if another of Thompson's groups, $T$ (which is a finitely presented simple group) is SCY, in this case with $b_{1}=0$ : that question partly motivated the present note.

From a geometer's viewpoint, Thompson's group $F$ has already been knocked out from the royalty of groups, i.e. Kähler groups, by the work of [17] (whose authors will hopefully condone us for the slight plagiarism in our title). However, as any finitely presented group, it keeps a footing as fundamental group of a symplectic 4-manifold, by [9] and, pushing the dimension up by 2 , of symplectic 6-manifolds with trivial canonical class by [7]. In spite of that, we will show that the constraints discussed above are sufficient to show that $F$ is not SCY. The main difficulty lies in the fact that the constraint on the first virtual Betti number, that is often very effective, is inconclusive:

Proposition. Thompson's group $F$ satisfies $b_{1}(F)=v b_{1}(F)=2$.

Proof. This is a consequence of the fact that ([4, Theorems 4.5]) the commutator subgroup $[F, F]$ is simple. Indeed, let $N \unlhd_{f} F$ be a finite index normal subgroup. Then $[N, N]$ is a normal subgroup of $[F, F]$. Since $[F, F]$ is simple (and, as $F$ is not virtually abelian, $N$ is not abelian) it follows that $[N, N]=[F, F]$. We therefore see that $H_{1}(N)=N /[N, N]=N /[F, F]$ is a subgroup of $F /[F, F] \cong \mathbb{Z}^{2}$. Now, as the Betti number is non decreasing on finite index subgroups, $b_{1}(N) \geq b_{1}(F)$. This entails that $H_{1}(N)$ is a finite index subgroup of $H_{1}(F)$, hence a copy of $\mathbb{Z}^{2}$ itself.

As the constraint on the virtual Betti number is inconclusive, we must resort to the Hausmann-Weinberger invariant $q(F)$ (whose calculation, to the authors' knowledge, has not appeared in the literature). While we are not able to calculate it exactly, we will show that it is strictly positive, whence $F$ is not a SCY group.

Theorem. The Hausmann-Weinberger invariant of Thompson's group F satisfies $0<q(F) \leq 2$. 
Proof. As is well known (see e.g. [6]) the Hausmann-Weinberger invariant satisfies the basic inequalities $2-2 b_{1}(F) \leq q(F) \leq 2-2 \operatorname{def}(F)$, where $\operatorname{def}(F)$ denotes the deficiency of $F$. The upper bound is easily obtained then from the fact that the presentation in (1) has deficiency 0 . To prove the lower bound, we will argue by contradiction. To start, we will compute the first $L^{2}$-Betti number. If $F$ were residually finite, the proposition, together with the Lück Approximation Theorem [15], would immediately imply its vanishing, but as this isn't the case one must argue differently. There is more than one way to proceed to this calculation (see [16, Theorem 7.10] for the original calculation, or [2, Theorem 1.8]). For the reader's benefit, we present the following, which is fairly explicit. Start with a well-known infinite presentation of the group $F$ :

$$
\left.F=\left\langle x_{0}, x_{1}, \ldots\right| x_{n} x_{i}=x_{i} x_{n+1}, \text { for all } 0 \leq i<n\right\rangle,
$$

that reduces to that in (1) putting $x_{n}=x_{0}^{1-n} x_{1} x_{0}^{n-1}$ for all $n \geq 2$. Defining the shift monomorphism $\phi: F \rightarrow F$ as $\phi\left(x_{i}\right)=x_{i+1}$ for all $i \geq 0$, the images $F(m)=$ $\phi^{m}(F)$ are isomorphic to $F$ itself, and $F$ is the properly ascending HNN-extension with base $F(1)$ itself, bonding subgroups $F(1)$ and $F(2)$ and stable letter $x_{0}$, i.e.

$$
F=\left\langle F(1), x_{0} \mid x_{0}^{-1} F(1) x_{0}=\phi(F(1))\right\rangle .
$$

As $F$ (hence $F(1), F(2)$ ) admits a finite presentation, the $L^{2}$-Betti number $b_{1}^{(2)}(F)$ vanishes by [12, Lemma 2.1]. As $F$ is an infinite group, $b_{0}^{(2)}(F)$ vanishes as well. Let $M$ be a 4-manifold with fundamental group $F$. By standard facts of $L^{2}$-invariants (see e.g. [16]) we have

$$
\chi(M)=2 b_{0}^{(2)}(F)-2 b_{1}^{(2)}(F)+b_{2}^{(2)}(M)=b_{2}^{(2)}(M) \geq 0,
$$

whence $q(F) \geq 0$. Assume then, by contradiction, that equality holds for some manifold $M$; by [6, Theorem 6] the only obstruction for $M$ to be an EilenbergMac Lane space $K(F, 1)$ is $H^{2}(F, \mathbb{Z}[F])$. Now for Thompson's group $F$ all cohomology groups $H^{*}(F, \mathbb{Z}[F])$ vanish ([10, Theorem 13.11.1]), so the obstruction vanishes; but in that case $F$ would be a Poincaré duality group of dimension 4, hence satisfy $H^{4}(F, \mathbb{Z}[F])=\mathbb{Z}$, that is false by the above.

We observe that the result above entails that the deficiency of $F$ is actually equal to zero. However, as the homology of $F$ is known (see e.g. [3]), this follows also from Morse inequality $\operatorname{def}(F) \leq b_{1}(F)-b_{2}(F)=0$ and the existence of the presentation of (1) of deficiency 0 .

Acknowledgment. We thank the referee for helpful comments. 


\section{References}

[1] S. Bauer, Almost complex 4-manifolds with vanishing first Chern class. J. Differential Geom. 79 (2008), no. 1, 25-32. Zbl 1144.57027 MR 2414748

[2] U. Bader, A. Furman, and R. Sauer, Weak notions of normality and vanishing up to rank in $L^{2}$-cohomology. Int. Math. Res. Not. IMRN 2014 (2014), no. 12, 3177-3189. Zbl 06340369 MR 3217658

[3] K. Brown, The homology of Richard Thompson's group F. In R. Grigorchuk, M. Mihalik, M. Sapir, and Z. Šuniḱ (eds.), Topological and asymptotic aspects of group theory. Contemporary Mathematics, 394. American Mathematical Society, Providence, R.I., 2006, 47-59. Zbl 1113.20043 MR 2216705

[4] J. Cannon, W. Floyd, and W. Parry, Introductory notes on Richard Thompson's groups. Enseign. Math. (2) 42 (1996), no. 3-4, 215-256. Zbl 0880.20027 MR 1426438

[5] S. Donaldson, Some problems in differential geometry and topology. Nonlinearity 21 (2008), no. 9, T157-T164. Zbl 1146.57301 MR 2430650

[6] B. Eckmann, 4-manifolds, group invariants, and $l_{2}$-Betti numbers. Enseign. Math. (2) 43 (1997), no. 3-4, 271-279. Zbl 0894.57002 MR 1489886

[7] J. Fine, D. Panov, The diversity of symplectic Calabi-Yau 6-manifolds. J. Topol. 6 (2013), no. 3, 644-658. Zbl 1288.14028 MR 3100885

[8] S. Friedl and S. Vidussi, On the topology of Symplectic Calabi-Yau 4-manifolds. J. Topol. 6 (2013), no. 4, 945-954. Zbl 1286.57023 MR 3145145

[9] R. Gompf, A new construction of symplectic manifolds. Ann. of Math. (2) 142 (1995), no. 3, 527-595. Zbl 0849.53027 MR 1356781

[10] R. Geoghegan, Topological methods in group theory. Graduate Texts in Mathematics, 243. Springer, New York, 2008. Zbl 1141.57001 MR 2365352

[11] J.-C. Haussmann and S. Weinberger, Caracteristiqués d'Euler et groupes fondamentaux des variétés de dimension 4. Comment. Math. Helv. 60 (1985), no. 1, 139-144. MR 0787667 Zbl 0566.57011

[12] J. A. Hillman, Four-manifolds, geometries and knots. Geometry \& Topology Monographs, 5. Geometry \& Topology Publications, Coventry, 2002. Zbl 1087.57015 MR 1943724

[13] T. J. Li, Symplectic 4-manifolds with Kodaira dimension zero. J. Differential Geom. 74 (2006), no. 2, 321-352. Zbl 1105.53068 MR 2259057

[14] T. J. Li, Quaternionic bundles and Betti numbers of symplectic 4-manifolds with Kodaira dimension zero. Int. Math. Res. Not. 2006 (2006), Article Id. 37385, 28 pp. Zbl 1129.57037 MR 2264722

[15] W. Lück, Approximating $L^{2}$-invariants by their finite-dimensional analogues. Geom. Funct. Anal. 23 (2013), no. 2, 622-663. Zbl 1273.22009 MR 3053758 
[16] W. Lück, $L^{2}$-invariants: theory and applications to geometry and $K$-theory. Ergebnisse der Mathematik und ihrer Grenzgebiete. 3. Folge, 44. Springer, Berlin, 2002. Zbl 1009.55001 MR 1926649

[17] T. Napier and M. Ramachandran, Thompson's group $F$ is not Kähler. In R. Grigorchuk, M. Mihalik, M. Sapir, and Z. Šuniḱ (eds.), Topological and asymptotic aspects of group theory. Contemporary Mathematics, 394. American Mathematical Society, Providence, R.I., 2006, 197-201. Zbl 1150.20031 MR 2216716

Received July 6, 2013

Stefan Friedl, Fakultät für Mathematik, Universität Regensburg, Germany

e-mail: sfriedl@gmail.com

Stefano Vidussi, Department of Mathematics, University of California, Riverside, CA 92521, U.S.A.

e-mail: svidussi@math.ucr.edu 